\author{
M. Reiger - V. Schwierzeck · C. Traidl-Hoffmann $n^{1,2,3}$ \\ 'Lehrstuhl und Institut für Umweltmedizin UNIKA-T, Technische Universität München und Helmholtz \\ Zentrum München, Augsburg, Deutschland \\ ${ }^{2}$ ZIEL - Institute for Food \& Health, Technische Universität München, Freising, Deutschland \\ ${ }^{3}$ Hochschulambulanz für Umweltmedizin, Universitätsklinikum Augsburg, Augsburg, Deutschland
}

\title{
Atopisches Ekzem und Mikrobiom
}

\begin{abstract}
Das kutane Mikrobiom ist ein Ökosystem, bestehend aus verschiedenen Mikroorganismen. Es bildet einen funktionellen Teil der Hautbarriere und steht in dauernder Interaktion mit dem kutanen Immunsystem. Durch moderne Sequenzierungsmethoden können wir erstmals Einblicke in die Gesamtheit des Mikrobioms und einzelner Bakterien gewinnen. Die Ergebnisse dieser Analysen und ihr Potenzial für neue Diagnostik-, Therapieund Präventionsstrategien werden von der Wissenschaft, aber auch von der Gesellschaft viel diskutiert. Der Artikel gibt eine Übersicht über die Bedeutung des Mikrobioms für das Krankheitsbild der Neurodermitis und soll dadurch auf neue Forschungsfelder hinweisen.
\end{abstract}

\section{Aktuelles zum atopischen Ekzem}

Das atopische Ekzem (AE, auch: atopische Dermatitis oder Neurodermitis) ist eine der häufigsten chronisch-entzündlichen Hauterkrankungen weltweit [1]. Bei Erwachsenen liegt die Prävalenz bei $2-8 \%$ und bei Kindern bei $20 \%$. Studien legen nahe, dass in Industrieländern die Prävalenz des AE und allgemein der Atopie über die letzten Jahrzehnte stetig gestiegen ist [2]. Die Gründe für die erhöhte Neigung zur Asthma- und Allergieentstehung sind noch vollständig $\mathrm{zu}$ klären; diskutiert werden auch Umweltfaktoren, z. B. eine fehlende Exposition gegenüber bakteriellen Bestandteilen [3].
Charakteristisch für das $\mathrm{AE}$ sind in Schüben verlaufende Ekzeme, die mit Hauttrockenheit und ausgeprägtem Juckreiz einhergehen. Der Juckreiz und die prominenten Hautentzündungen üben einen hohen psychosozialen Druck auf Betroffene und ihre Angehörigen aus [4]. Daraus resultieren hohe Kosten für das Gesundheitssystem. Die Neurodermitis ist häufig mit anderen Erkrankungen des atopischen Formenkreises wie Allergie, Rhinokonjunktivitis und Asthma bronchiale assoziiert [5]. Eine gestörte Hautbarriere gilt dabei als Eintrittspforte für Allergene und hat einen großen Anteil an der Pathogenese des AE. Die Vorstellung, dass das AE ein späteres Auftreten von IgE-assoziierten, atopischen Erkrankungen begünstigt, wird auch als „atopischer Marsch“ bezeichnet [1].

I) Das heterogene Bild der AE mit variablen Endotypen weist auf unterschiedliche Pathomechanismen hin

Die Neurodermitis ist eine dynamische Erkrankung mit einem klinischen Bild, das bezüglich der Manifestationsstellen altersabhängig ist; sie kann stark schwanken in Ausprägung und klinischem Erscheinungsbild. Bei Säuglingen ist häufig zuerst die Kopfhaut betroffen, dann folgen weitere Hautareale. Bei Schulkindern und Erwachsenen manifestiert sich $\mathrm{AE}$ meist an den Beugeseiten der Extremitäten, im Kopf-Hals-Bereich oder an den Händen. Das heterogene Krankheitsbild des AE mit unterschiedlichen Endotypen ist Hinweis darauf, dass der Erkrankung unterschiedliche Pathomechanismen zugrunde liegen [6, 7]. Ein Ziel der Forschung ist es, diese Mechanismen (genetische Prädisposition, Umweltfaktoren, Hautbarrierestörung, Inflammation und Dysbiose) auf molekularer Ebene zu verstehen, um in Zukunft eine individuelle, personalisierte Therapie anwenden zu können. Die Therapie der Neurodermitis hat deshalb in den letzten Jahren eine revolutionäre Entwicklung erfahren, die sich auch in den nächsten Jahren fortsetzen wird. Ähnlich wie bei der Psoriasis haben Biologika als Systemtherapie in das Management der Neurodermitis Einzug gehalten und verbessern neben den klinischen Symptomen vor allem die Lebens-

\begin{tabular}{|ll}
\hline Abkürzungen \\
\hline$A E$ & $\begin{array}{l}\text { Atopisches Ekzem, auch: atopische } \\
\text { Dermatitis oder Neurodermitis }\end{array}$ \\
\hline AMP & Antimikrobielle Peptide \\
\hline APZ & Antigenpräsentierende Zellen \\
\hline HPV & Humanes Papillomavirus \\
\hline Ig & Immunglobulin(e) \\
\hline IL & Interleukin(e) \\
\hline NMF & Natürliche Feuchtigkeitsfaktoren \\
\hline 16S rDNA & 16S ribosomale DNA \\
\hline S. aureus & Staphylococcus aureus \\
\hline $\begin{array}{l}\text { S. epider- } \\
\text { midis }\end{array}$ & Staphylococcus epidermidis \\
\hline S. hominis & Staphylococcus hominis \\
\hline TJ & "Tight junctions" \\
\hline TLR & Toll-like-Rezeptor \\
\hline
\end{tabular}




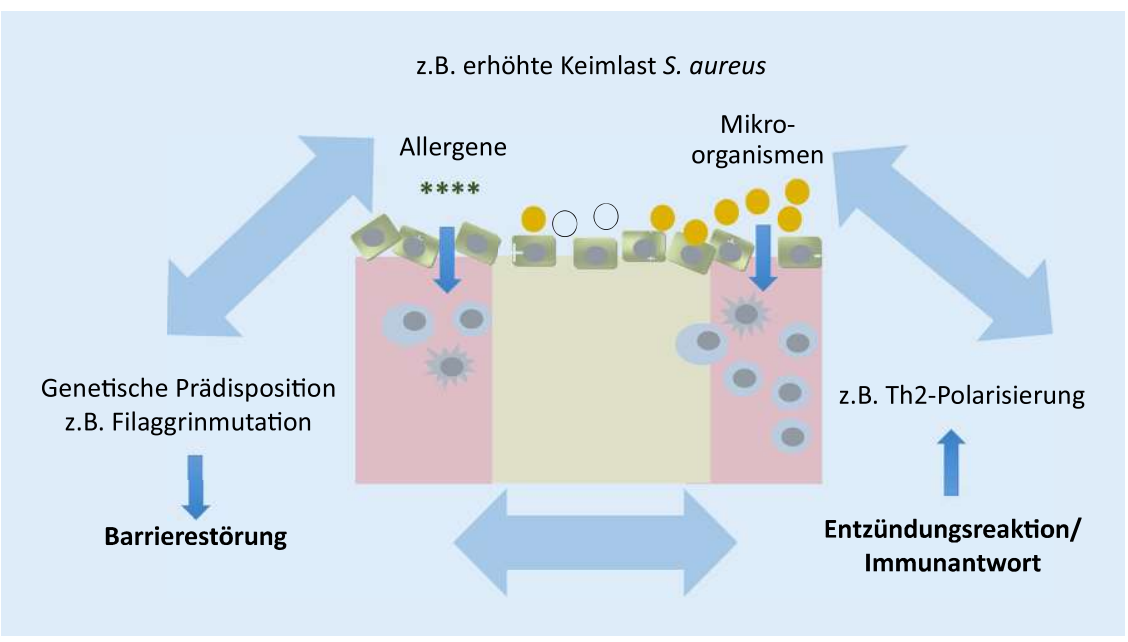

Abb. $1 \Delta$ Die Physiologie der Haut ist durch eine interaktive Dreiecksbeziehung zwischen Hautbarriere, bakterieller Mikroflora und dem lokalen Immunsystem gekennzeichnet. Bei der Neurodermitis ist dieses System aus dem Gleichgewicht geraten

qualität der Betroffenen [8-10]. Neueste Forschungsergebnisse liefern bereits Hinweise auf die positive Beeinflussung des Mikrobioms durch Biologika.

\section{Homöostase der Haut: Interaktion zwischen Epithel, Mikrobiom und Immunsystem}

Die Haut ist unser größtes Körperorgan. Sie schützt uns nach außen vor Einflüssen der Umwelt und vor Krankheitserregern. Aber die Haut ist nicht nur ein "Schutzmantel“ oder eine rein physikalische Barriere. Tatsächlich besteht eine dynamische Interaktion zwischen Hautbarriere, Mikrobiom und dem menschlichen Immunsystem (• Abb. 1; [11]).

Die Hautbarriere bietet zugleich physikalischen, chemischen und immunologischen Schutz gegen Umwelteinflüsse. Auch das Mikrobiom ist Teil der Hautbarriere und ein wichtiger Interaktionspartner mit den anderen Komponenten dieser Schutzschicht. Die äußerste Hautschicht, das Stratum corneum, bietet durch die Verhornung Schutz gegen mechanische Störungen. Im angrenzenden Stratum granulosum bilden die Keratinozyten zusammen mit den TJ eine dichte physikalische Barriere aus. TJ-Proteine sind in der Regel transmembranär. Wichtige Beispiele sind die Proteine Claudin, Occludin und Zona occludens. Teil der chemischen Barriere sind Faktoren, die zum Erhalt des sauren pH-Wertes und dem Feuchtigkeitsgehalt der Hautoberfläche beitragen [12]. Die NMF beinhalten verschiedene Bestandteile, wie Aminosäuren, Laktat, Elektrolyte und freie Fettsäuren. Diese entstehen größtenteils, wenn in den äuBersten Schichten des Stratum corneum Filaggrin zu freien Aminosäuren, Urocaninsäure und Pyrrolidoncarbonsäure abgebaut wird. Eine starke genetische Assoziation zu AE entsteht durch eine Mutation im Filaggrin-Gen, die einen Funktionsverlust des Gens zur Folge hat. Man nimmt an, dass bei ungefähr einem Drittel aller europäischen AE-Patienten eine Mutation im Filaggrin-Gen vorliegt. Bei AE-Patienten kann eine Besiedlung durch S. aureus die Hautbarriere zusätzlich, d.h. auch bei Patienten ohne Mutation im Filaggrin-Gen, stören, indem es die Barriereproteine Filaggrin und Loricrin herabreguliert und durch die Sekretion von bakteriellen Proteasen die Hautbarriere direkt angreift. a-Toxin ist ein typischer Virulenzfaktor von $S$. aureus mit hämolytischer und zellschädigender Wirkung. Viele S.-aureus-Stämme verfügen über zusätzliche Pathogenitätsfaktoren, die meisten von ihnen über Exotoxine mit Superantigeneigenschaften, wie etwa Enterotoxin A und B. Die Sekretion dieser Toxine kann die Schädigung der Hautbarriere weiter verstärken [13].

Das kutane Mikrobiom ist ein Ökosystem verschiedenster Mikroorganismen, welche die Hautoberfläche besiedeln. Diese Gemeinschaft ist relativ stabil. Dadurch gelingt es den kommensalen Bakterien, potenzielle Pathogene fernzuhalten. Gleichzeitig trainiert die Interaktion mit kommensalen Bakterien unser Immunsystem und bereitet es auf einen Angriff durch Krankheitserreger vor. Beispielsweise induziert das kommensale Bakterium S. epidermidis in menschlichen Keratinozyten die Produktion von AMPs [14] und ist in der Lage, auch selbst AMPs zu produzieren, um sich vor umgebenden Mikroben zu schützen. Studien konnten auch zeigen, dass der bei Atopikern häufigere Typ 2 der Helferzellen die Abwehrfähigkeit der Hautbarriere schwächt. Experimentell konnte nachgewiesen werden, dass klassische Th2(„T helper“)-Botenstoffe wie IL 4 oder IL 13 die IL-17-induzierte Sekretion von AMPs inhibieren können [15].

\section{》) Vermindert sezernierte oder funktionell eingeschränkte AMPs schwächen die Hautbarriere strukturell}

Die Haut ist eines der größten Immunorgane des Körpers. Das Immunsystem der Haut besteht sowohl aus Komponenten des angeborenen als auch aus Komponenten des erworbenen Immunsystems [11]. In der Epidermis sind LangerhansZellen lokalisiert, eine Untergruppe der APZs, die nur in der Haut vorkommen. Langerhans-Zellen nehmen effizient Proteinantigene auf und sind in der Lage, lokal ein Th2-Milieu zu induzieren. In der Dermis sind dendritische Zellen, Mastzellen und T-Zellen ansässig. Schätzungen gehen davon aus, dass in der Hautoberfläche eines gesunden Erwachsenen etwa $20 \mathrm{Mrd}$. T-Zellen angesiedelt sind [16]; zu diesen gehören auch relativ seltene Subpopulationen wie $\gamma \delta$-T-Zellen und NKT(„,natural killer T“)-Zellen. Ein Kennzeichen für die Immunpathologie in AE-Patienten ist deren übermäßige Th2Immunantwort. Die gesteigerte Produktion von Th2-Zytokinen hemmt die Produktion von AMPs in der Haut $[15,17]$. AMPs verhindern bei Hautgesunden ein 
übermäßiges Wachstum von S. aureus. Die verminderte Sezernierung oder eine gestörte Funktion von AMPs bei AEPatienten trägt zur Dysbiose des Hautmikrobioms bei und schwächt die Hautbarriere zusätzlich strukturell [18]. Dadurch sind AE-Patienten anfälliger für Hautinfektionen, z. B. eine Impetiginisierung durch $S$. aureus und Infektionen mit Viren der Art Herpes simplex, Molluscum contagiosum oder Coxsackie [19, 20].

\section{Das Hautmikrobiom}

Die menschliche Haut ist der Lebensraum für eine Vielzahl von Bakterien, Viren und Pilzen. Die Gesamtheit dieser Mikroorganismen hinsichtlich ihrer Vielfalt und Anzahl bezeichnet man als das Mikrobiom der Haut. Grundsätzlich stellt die Haut eine feindliche Umgebung für Bakterien dar: trocken, kühl, saures Milieu und konstant verschiedene Umwelteinflüsse. Aus diesem Grund dominieren auf der Haut grampositive Bakterien, die wegen ihrer an Peptidoglykanen reichen Zellwand widerstandsfähiger sind. Trotz dieser Wachstumsbedingungen leben auf unserer Haut ca. 10.000 Bakterien $/ \mathrm{cm}^{2}$. Bei der Mehrheit der Bakterien aufder Haut handelt es sich um sog. Kommensalen (abgeleitet vom Lateinischen cum mensa, etwa: Tischgenossen), die mit ihrem Wirt eine friedliche, zum Teil symbiotische Wechselbeziehung eingehen. Nur wenige Hautbakterien haben aufgrund ihrer Pathogenitätsfaktoren eine Bedeutung als Krankheitserreger, beispielsweise S. aureus, einer der häufigsten Erreger von ambulanten und nosokomialen Weichteilinfektionen.

Da das Hautmilieu je nach Körperregion verschieden ist, unterscheidet sich selbst bei Hautgesunden die bakterielle Besiedlung der Haut je nach Lokalisation. Auch Alter und Geschlecht beeinflussen das Hautmikrobiom. Feuchte Hautbereiche, z. B. Achselhöhlen, sind vor allem mit Staphylokokken und Corynebakterien besiedelt. Auf fettigen Hautregionen wie Stirn und Rücken finden sich Propionibakterien, deren Spezies nach moderner Nomenklatur Cutibacterium genannt wird. Ihr wohl bekanntester Vertreter, das Cutibacterium acnes, ist in der Lage, Triglyzeride zu spalten, womit es zum Erhalt

\section{Reiger · V. Schwierzeck · C. Traidl-Hoffmann \\ Atopisches Ekzem und Mikrobiom}

\section{Zusammenfassung}

Hintergrund. Neurodermitis ist eine chronisch-entzündliche Hauterkrankung, die durch Hautbarrierestörung, Entzündung und Dysbiose gekennzeichnet ist. Darüber hinaus ist das atopische Ekzem mit anderen Krankheiten des atopischen Formenkreises wie Allergie, Rhinokonjunktivitis und Asthma assoziiert. Das Mikrobiom der Haut besteht aus Bakterien, Viren und Pilzen. Bei Patienten mit Neurodermitis ist häufig ein Ungleichgewicht des Mikrobioms (Dysbiose) feststellbar.

Fragestellung. Noch ist nicht vollständig geklärt, welchen Einfluss die Dysbiose und das kutane Mikrobiom auf die Entstehung und den Verlauf des atopischen Ekzems haben. Mittels moderner Sequenzierungsmethoden soll nun die Rolle des Hautmikrobioms bei der Pathogenese der Neurodermitis entschlüsselt werden.

Material und Methoden. Vorgestellt und diskutiert werden Ergebnisse aktueller Grundlagenarbeiten.

\section{Atopic eczema and microbiome}

\section{Abstract}

Background. Atopic eczema is a chronic inflammatory skin disease characterized by skin barrier disruption, inflammation and dysbiosis. Furthermore, atopic eczema is associated with other diseases of the atopic group, such as allergies, rhinoconjunctivitis and asthma. The skin microbiome consists of bacteria, viruses and fungi. Patients suffering from atopic eczema often show an imbalance (dysbiosis) of the microbiome.

Objective. It is not yet completely clarified what influence dysbiosis and the cutaneous microbiome have on the development and severity of atopic eczema. Modern sequencing methods will be used to investigate the role of the skin microbiome in the pathogenesis of atopic eczema in the future.

Material and methods. This article presents and discusses the results of current basic research.

Results. The human skin microbiome differs according to body region, age and
Ergebnisse. Das Hautmikrobiom unterscheidet sich nach Körperregion, Alter und Geschlecht und steht in ständigem Austausch mit der Hautbarriere und dem kutanen Immunsystem. Bei der Neurodermitis entsteht eine Dysbiose mit einer erhöhten Keimlast von Staphylococcus aureus und einer Verringerung von kommensalen Hautbakterien. Das veränderte Mikrobiom bei Neurodermitis kann somit auch die Hautbarriere und Entzündungsreaktionen beeinflussen.

Schlussfolgerungen. Das Verständnis des Hautmikrobioms hat sich in den letzten Jahren verbessert. Zweifellos wird davon auch das Verständnis der Pathogenese der Neurodermitis profitieren. Zugleich können diese Erkenntnisse in Zukunft die Grundlage für neue Therapie- und Präventionsstrategien bilden.

\section{Schlüsselwörter}

Dysbiose - Mikrobiota - Inflammation . Immunsystem · Allergien

gender. It interacts with the skin barrier and the cutaneous immune system. Patients suffering from atopic eczema develop dysbiosis consisting of an increased load of Staphylococcus aureus and a reduction of commensal skin bacteria. The altered skin microbiome in patients suffering from atopic eczema may also affect skin barrier function and inflammatory reactions.

Conclusion. Knowledge of the skin microbiome has improved in recent years. This will certainly improve the understanding of the pathogenesis causing atopic eczema. These findings may also form the foundation of new treatment and prevention strategies for atopic eczema in the future.

Keywords Dysbiosis · Microbiota · Inflammation . Immune system · Allergies 

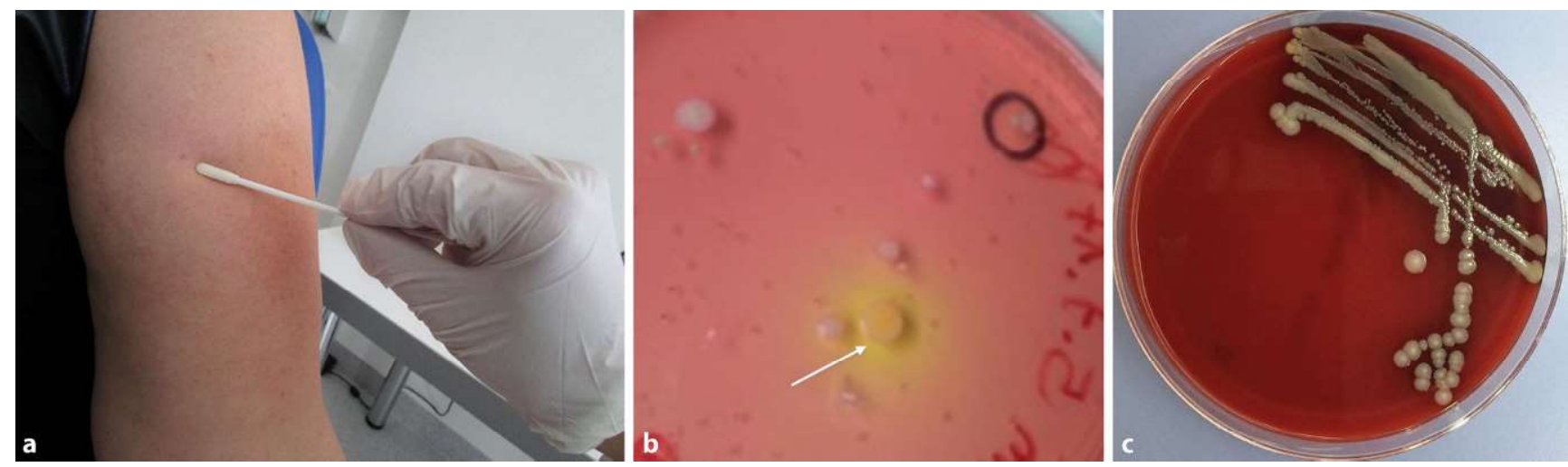

Abb. $2 \Delta$ Entnahme eines Mikrobiomabstrichs mit speziellen Abstrichtupfern, die frei von bakterieller DNA sind (a). Kultivierung von Hautbakterien auf Indikatorplatten (z. B. Mannit-Kochsalz-Platte), ermöglicht die schnelle Identifizierung von S. aureus (weißer Pfeil, b). Für funktionelle Studien werden ausgewählte Bakterienstämme isoliert und weiter untersucht (c)

des sauren $\mathrm{pH}$-Wertes der Haut beiträgt. Interessanterweise ist die Besiedlung mit C. acnes auf läsionaler Haut geringer als bei gesunden Kontrollen und korreliert invers mit der Besiedlung durch S. aureus.

Bei den Spezies der Staphylokokken unterscheidet man zwischen den koagulasenegativen und koagulasepositiven Spezies. Koagulasenegative Staphylokokken, wie S. epidermidis und S. hominis, sind typische Kommensalen. S. aureus ist koagulasepositiv. Schon lange ist bekannt, dass bei Neurodermitispatienten eine Dysbiose beim Mikrobiom der Haut feststellbar ist. Im akuten Schub nimmt die Keimlast von $S$. aureus zu, und Läsionen sind häufig mit $S$. aureus kolonisiert.

Auch Pilze sind Teil des menschlichen Hautmikrobioms. Man verwendet hier auch den Begriff Mykobiom. Die meisten Körperoberflächen sind mit Pilzen besiedelt, die häufigsten Spezies sind Malassezia, Candida, Aspergillus und Penicillium.

Bis vor ungefähr 10 Jahren beruhte unser Wissen über das menschliche Mikrobiom auf mikrobiologischen Kulturergebnissen. Die Mikrobiologie arbeitete agarbasiert, hauptsächlich anzüchtbare Bakterien konnten darüber identifiziert und quantifiziert werden. Die Verbesserung von genomischen Sequenzierungsmethoden - das "next generation sequencing" - revolutionierte die Erforschung des Mikrobioms. 16S-rDNA-Sequenzierung, z. B. im Rahmen des Human Microbiome Project, ermöglichte erstmals Einblicke in die gesamte Vielfalt des menschlichen bakteriellen Mikrobioms [21]. Zunächst war bei vielen Forschungsprojekten das Darmmikrobiom der Untersuchungsgegenstand. Wenig später nutzte man die Analysemethoden, um das Mikrobiom der Haut bei den Erkrankungen zu untersuchen, für die eine mikrobielle Dysbiose vermutet wurde, z. B. bei der Neurodermitis [22]. Eine Herausforderung dabei war, geeignete Verfahrensweisen für die Hautmikrobiomanalyse zu etablieren, z.B. wie Proben des Hautmikrobioms am besten zu gewinnen seien (• Abb. 2; [23]). AuBerdem enthalten die Proben des Hautmikrobioms im Vergleich zu denen des Darmmikrobioms nur geringe Mengen an bakterieller DNA, was bei der Sequenzierung und Analyse der Ergebnisse zu Schwierigkeiten führen kann. Analog zum Mikrobiom werden genomische Sequenzierungsmethoden auch zur Analyse des Mykobioms verwendet.

\section{Hautmikrobiomanalysen - neue Erkenntnisse für $\mathrm{AE}$}

Das immer detailliertere Wissen über die Mikroflora der Haut und deren räumliche und zeitliche Entwicklung bildet die Grundlage für neue Therapieansätze und neue Präventionskonzepte.

Studien zeigen, dass insbesondere die Zunahme des Pathogens S. aureus mit einer Verschlechterung des Hautzustandes von Patienten mit Neurodermitis einhergeht. Die Veränderung im Gleichgewicht der Mikroorganismen wird mit einer strukturellen Veränderung der Barriereschichten und des Immunsystems assoziiert (• Abb. 3; [24]). Im komplexen System der Interaktion zwischen den Mikroorganismen untereinander sowie zwischen Mikroorganismen, Barriereschichten und Immunsystem bleibt zu klären, ob es kausale Zusammenhänge für ihre Veränderungen in der Interaktion gibt [11].

Viele Mikroorganismen der Haut sekretieren Substanzen, die eine Besiedlung ihrer speziellen Nische durch andere Mikroben verhindern oder auch deren konkurrierendes Verhalten beeinflussen [25]. Generell ist das Mikrobiom im Erwachsenenalter relativ stabil, solange sich nicht die Umgebung oder Umwelt merklich ändert. Dabei helfen Mikroorganismen bei der Ausbildung der angeborenen (innaten) und der adaptiven Immunantwort der Hautbarriere [26]. Verschiedene aktuelle Studien zeigen, dass kommensalen Bakterien eine wichtige Funktion in der Zurückhaltung von S. aureus zukommt, indem sie, wie etwa $S$. hominis oder S. lugdunensis, spezifische antimikrobielle Substanzen produzieren [25].

Das Mikrobiom im Kleinkindesalter ist dabei von besonderer Wichtigkeit. Eine Studie zeigt, dass die Besiedlung der Haut mit kommensalen Staphylokokken innerhalb der ersten Lebensmonate mit einem niedrigeren Risiko verbunden ist, im Alter von einem Jahr an am AE zu erkranken [27]. Entsprechend konnte eine andere Studie zeigen, dass Kleinkinder, die im Alter von 3 Monaten mit S. aureus besiedelt waren, ein erhöhtes Risiko ha- 


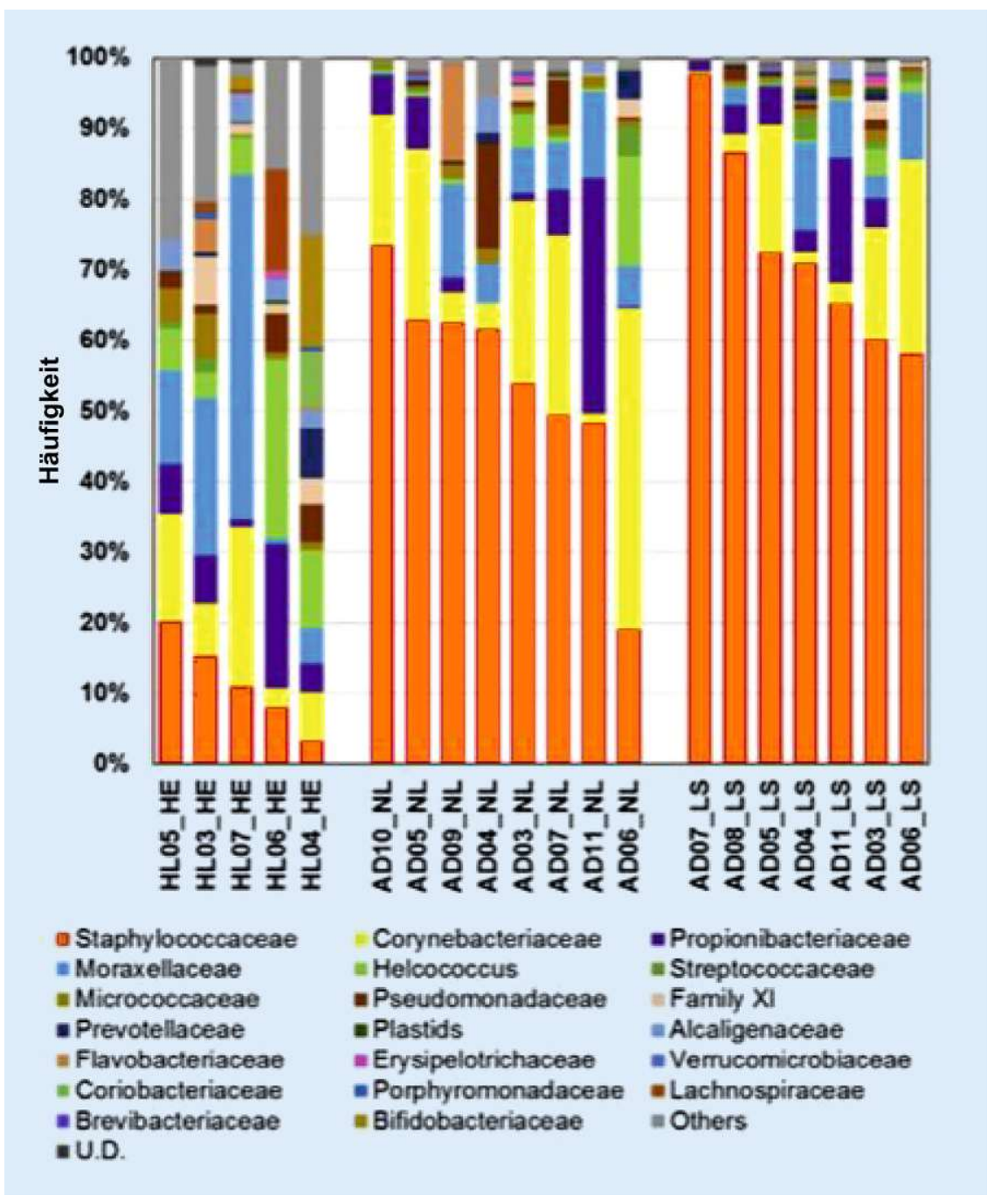

Abb. 3 ॥ Mikrobiom Taxonomie auf Familienebene. Mit der Mikrobiomanalyse lassen sich Bakterienspezies identifizieren, wenn das Gleichgewicht der bakteriellen Flora gestört ist. Bei Gesunden $(H E)$ ist das Hautmikrobiom von verschiedenen Bakterienspezies besiedelt, bei Neurodermitispatienten (AD-NL [atopische Dermatitis, läsionale Haut], $A D-L S$ [atopische Dermatitis, nichtläsionale Haut]) nimmt die bakterielle Vielfalt ab. Dies ist bei nichtläsionaler Haut $(N L)$ bemerkbar, bei läsionaler Haut (LS) besonders ausgeprägt. (Mod. nach [23])

ben, bis zu ihrem zweiten Lebensjahr an AE zu erkranken [28]. Die Einnahme von Antibiotika in den ersten beiden Lebensjahren ist ein bekannter Einflussfaktor, der das Risiko einer Neurodermitiserkrankung erhöht. Der Kontakt zu einer möglichst diversen Umwelt, wie z. B. der Kontakt zu Hunden oder das Aufwachsen auf einem Bauernhof, verringert das Risiko, an Neurodermitis zu erkranken [3, 29, 30]. Umstritten ist der Einfluss der Art der Geburt (Spontangeburt oder Kaiserschnitt), da hier die aktuelle Studienlage unterschiedliche Ergebnisse präsentiert.

Neben den Bakterien besiedeln Pilze der Gattung Malassezia die Haut und sind zyten sowie die Induktion autoreaktiver T-Zellen [32].

Immer größere Bedeutung wird inzwischen den Viren auf der Haut beigemessen, die mangels konservierter Regionen nur verhältnismäßig schwer und mit großem Aufwand in ihrer Gesamtheit als sog. Virom erfasst werden können. Dennoch beschäftigen sich einige Forschergruppen mit den Viren, z. B. mit dem HPV, dem Polyomavirus oder den Bakteriophagen von Propionibakterien und Staphylokokken. Bakteriophagen zeichnen sich durch eine hohe Variabilität aus und können als Vektoren für die Anpassung von ihren Wirtsbakterien dienen [33]. Es ist gut belegt, dass Neurodermitispatienten häufig unter viralen Infektionen mit Molluscum contagiosum oder Papillomaviren leiden. Besonders dramatisch sind schwere, generalisierte Verläufe einer Herpes-simplex-Typ1-Infektion mit einem konsekutiven Eczema herpeticum, das auch zu lebensbedrohlichen Komplikationen führen kann. Verschiedene Studien konnten beim $\mathrm{AE}$ eine verminderte antivirale Interferon- $\gamma$-Immunantwort feststellen. T-Zell-Linien von AE-Patienten reagierten auf die Stimulation mit InfluenzaAntigenen durch eine Reduktion der Interferon- $\gamma$-Produktion. Die Ausschüttung von Th2-Zytokinen, wie IL 4, war dagegen erhöht [20].

\section{》) Die Besiedlung des Darmes mit Bifidobakterien korreliert negativ mit dem Schweregrad des $A E$}

Teil der Normalflora. Interessanterweise wurden in läsionalen Hautstellen mehr Malassezia-DNA als in gesunden nachgewiesen, damit wurde ein Zusammenhang mit der symptomatischen Ausprägung der Krankheit festgestellt. Genauere Untersuchungen haben gezeigt, dass bei Gesunden und Patienten unterschiedliche Stämme auftreten. Dies weist auf (mindestens) einen pathogenen Stamm hin, der an der Verschlechterung des AE beteiligt ist [31]. Mögliche Pathogenitätsmechanismen sind die Induktion proinflammatorischer Zytokine, die Expression des TLR2 und des TLR4 auf Keratino-
Einflüsse, die das Hautmikrobiom beeinträchtigen, sind - neben persönlichen Gewohnheiten wie Häufigkeit und Art von Desinfektion, Verwendung von Seife und Cremes - Umweltfaktoren, wie beispielsweise ultraviolette Strahlung und Luftfeuchtigkeit, aber auch psychischer Stress $[4,34,35]$. So konnten verschiedene Studien zeigen, dass im Kindesalter die Benutzung von Pflegecremes Einfluss auf die Hautbakterien hatte und bei Kindern mit einer familiären Veranlagung für Neurodermitis zur erhöhten Diversität des Mikrobioms führte. 


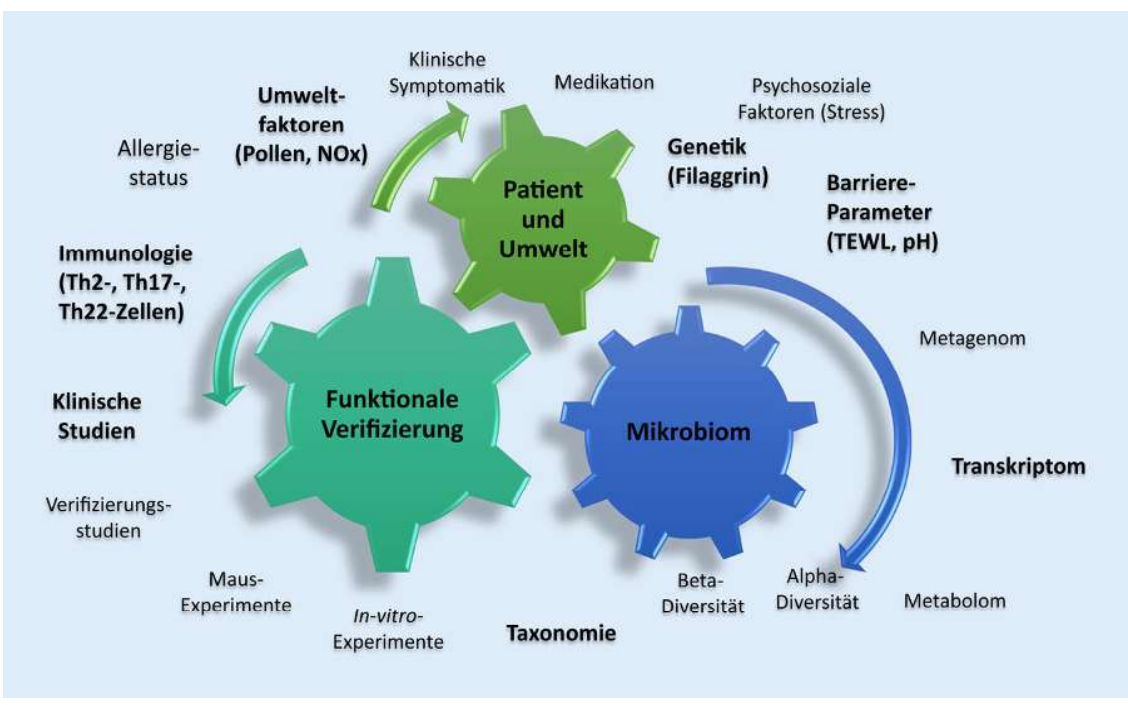

Abb. $4 \Delta$ Bei der Neurodermitis ist das Gleichgewicht aus physiologischer Barriere, lokalem Immunsystem und dem Mikrobiom gestört, sodass die Haut vermehrt Umwelteinflüssen ausgesetzt ist. Zum Verständnis dieser komplexen Zusammenhänge sind detaillierte Studien nötig, die verschiedene Einflussfaktoren mit der klinischen Symptomatik korrelieren

Inwieweit es eine Interaktion zwischen Darm und Haut gibt, wird aktuell erforscht. Es verdichten sich die Hinweise, dass Darm- und Hautmikrobiom Ansatzpunkte für die Behandlung von $\mathrm{AE}$ bieten. Beispielsweise konnte gezeigt werden, dass der Anteil an Bifidobakterien im Darm von Neurodermitispatienten im Vergleich zu Gesunden geringer ist. Die Besiedlung des Darmes mit diesen Bakterien ist negativ mit dem Schweregrad der Neurodermitis korreliert [36].

\section{Hautmikrobiomanalyse - Quo vadis?}

Durch moderne Sequenzierungsmethoden konnten zusätzliche Erkenntnisse über das Hautmikrobiom gewonnen werden. Die Mikrobiomanalyse erlaubt jedoch nur eine Identifikation der Mikroorganismen. Neue Methoden müssen erforscht werden, um die Funktionsweise und die Pathogenitätsfaktoren der Bakterien zu untersuchen. Da die Dysbiose der Hautflora bei AE-Patienten durch eine vermehrte Besiedlung durch S. aureus gekennzeichnet ist, haben sich bereits einige Studien mit der Charakterisierung von $S$. aureus beschäftigt. Es konnte nachgewiesen werden, dass bei AE-Patienten mit schwerem Symptomverlauf eine klonale Expansion von S.-aureusStämmen stattfindet [37]. Außerdem tor zu identifizieren, der das richtige Ziel darstellt. Vermutlich ist deshalb ein multifaktorieller Ansatz der richtige Weg.

Aktuell wird die Therapie des AE durch neue Medikamente wie Biologika und antiinflammatorisch aktive Wirkstoffe regelrecht revolutioniert. Langfristig wird die Herausforderung für Hautärzte sein, Patienten mit Neurodermitis eine individualisierte Therapie anbieten zu können. Dies erfordert ein Verständnis über die Pathomechanismen, die den unterschiedlichen Endotypen und Schweregraden der AEErkrankung zugrunde liegen [7]. Dazu müssen der Zusammenhang zwischen Hautbarrierestörung, Inflammation und Dysbiose bei Patienten mit AE detailliert untersucht und sowohl mit klinischen Symptomen als auch mit Umweltfaktoren korreliert werden (• Abb. 4). $\mathrm{Zu}$ erforschen, ob bzw. wie das Mikrobiom durch Biologika verändert werden kann und ob diese Veränderung zur Wirkungsfähigkeit dieser Medikamente beiträgt, ist deshalb ein wichtiges Ziel.

Die Haut als Grenzorgan zwischen Mensch und Umwelt bildet eine robuste Barriere, die im Falle eines Gleichgewichts zwischen der physiologischer Barriere, dem lokalen Immunsystem und den Mikroorgansimen vor verschiedensten Umwelteinflüssen schützt. Wenn, wie bei der AE, ein Ungleichgewicht besteht, wird die Barrierefunktion gestört, die Haut wird durchlässig für Umwelteinflüsse, Mikroorganismen und Allergene. Eine Behandlung der AE kann somit auch eine präventive Wirkung auf die Entwicklung von Allergien ausüben.

\section{\) Die Behandlung der AE kann auch zur Prävention von Allergien beitragen}

Die Wiederherstellung der Hautbarrierefunktion bei Patienten mit Neurodermitis kann auf verschiedene Arten unterstützt werden. Die Interaktion zwischen Mensch und Mikrobiom ist dabei ein wichtiger Ansatzpunkt. Aktuelle und zukünftige Studien werden dazu beitragen, die Komplexität dieser Interaktion besser $\mathrm{zu}$ verstehen und neue, effektivere Therapien zu entwickeln. 


\section{Fazit für die Praxis}

- Das Mikrobiom der Haut umfasst Bakterien, Viren und Pilze und trägt selbst zum Erhalt des kutanen Immunsystems und der Hautbarriere bei.

- Neurodermitis ist durch eine krankheitsverstärkende Wiederkehr aus Hautbarrierestörung, Inflammation und Dysbiose gekennzeichnet.

- Die Dysbiose besteht aus einer erhöhten Keimlast mit S. aureus bei gleichzeitiger Verringerung kommensaler Hautbakterien. Moderne Sequenzierungsmethoden erlauben, das gesamte Mikrobiom zu identifizieren und einzelne Bakterienstämme zu charakterisieren. Diese Ergebnisse müssen mit weiteren Parametern, wie klinischer Symptomatik, Immunantwort oder Funktionalität der Hautbarriere, korreliert und mit funktionalen Studien bestätigt werden.

- Ziel dieser Forschungsansätze ist, die Pathomechanismen des atopischen Ekzems (AE) besser zu verstehen, um eine für den Patienten individualisierte Therapie auszuwählen.

- Ziel der Therapie ist vor allem die Stärkung der Hautbarriere, um der Entwicklung von Allergien und Asthmaerkrankungen frühzeitig vorzubeugen. Der Einsatz von Prä-, Pro- und Symbiotika zur Korrektur der Dysbiose beim AE ist zwar ein möglicher Ansatz, aber die genauen Wirkmechanismen dieser Präparate sind noch unklar.

\section{Korrespondenzadresse}

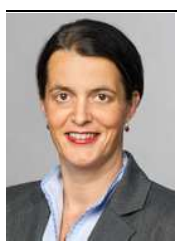

Univ.-Prof. Dr. med. C. Traidl-Hoffmann Lehrstuhl und Institut für Umweltmedizin UNIKA-T, Technische Universität München und Helmholtz Zentrum München Neusäßer Str. 47, 86156 Augsburg, Deutschland c.traidl-hoffmann@tum.de

\section{Einhaltung ethischer Richtlinien}

Interessenkonflikt. C. Traidl-Hoffmann war als Beraterin und Referentin gegen Honorar tätig für Sanofi, Novartis, Töpfer, Sebapharma bzw. erhielt Forschungsgelder von Sebapharma, Danone Nutricia, Töpfer. M. Reiger und V. Schwierzeck geben an, dass kein Interessenkonflikt besteht

Für diesen Beitrag wurden von den Autoren keine Studien an Menschen oder Tieren durchgeführt. Für die aufgeführten Studien gelten die jeweils dort angegebenen ethischen Richtlinien.

\section{Literatur}

1. Weidinger $S$, Beck $L A$, Bieber $T$, Kabashima K, Irvine AD (2018) Atopic dermatitis. Nat Rev Dis Primers $4(1): 1$

2. Mallol J, Crane J, von Mutius E, Odhiambo J, Keil U, Stewart A et al (2013) The International Study of Asthma and Allergies in Childhood (ISAAC) Phase Three: a global synthesis. Allergol Immunopathol (madr) 41(2):73-85

3. von Mutius E, Braun-Fahrlander C, Schierl R, Riedler J, Ehlermann S, Maisch S et al (2000) Exposure to endotoxin or other bacterial components might protect against the development of atopy. Clin Exp Allergy 30(9):1230-1234

4. Drucker AM, Wang AR, Li WQ, Sevetson E, Block JK, Qureshi AA (2017) The burden of atopic dermatitis: summary of a report for the national eczema association. JInvest Dermatol 137(1):26-30

5. Ferreira MA, VonkJM, Baurecht H, Marenholz I, Tian C, Hoffman JD et al (2017) Shared genetic origin of asthma, hay fever and eczema elucidates allergic disease biology. Nat Genet 49(12):1752-1757

6. Bieber T, D'Erme AM, Akdis CA, Traidl-Hoffmann C, Lauener R, Schappi G et al (2017) Clinical phenotypes and endophenotypes of atopic dermatitis: where are we, and where should we go? J Allergy Clin Immunol 139(4S):58-64

7. Czarnowicki T, He H, Krueger JG, GuttmanYassky E (2019) Atopic dermatitis endotypes and implications for targeted therapeutics. J Allergy Clin Immunol 143(1):1-11

8. Simpson EL, Bruin-Weller M, Flohr C, Ardern-Jones MR, Barbarot S, Deleuran M et al (2017) When does atopic dermatitis warrant systemic therapy? Recommendations from an expert panel of the International Eczema Council. J Am Acad Dermato 77(4):623-633

9. Brunner PM, Pavel AB, Khattri S, Leonard A, Malik $\mathrm{K}$, Rose $S$ et al (2019) Baseline IL-22 expression in patients with atopic dermatitis stratifies tissue responses to fezakinumab. J Allergy Clin Immunol 143(1):142-154

10. Guttman-Yassky E, Bissonnette R, Ungar B, SuarezFarinas M, Ardeleanu M, Esaki $\mathrm{H}$ et al (2019) Dupilumab progressively improves systemic and cutaneous abnormalities in patients with atopic dermatitis. J Allergy Clin Immunol 143(1):155-172

11. Eyerich S, Eyerich K, Traidl-Hoffmann C, Biedermann T (2018) Cutaneous barriers and skin immunity: differentiating a connected network. Trends Immunol 39(4):315-327

12. Rippke F, Schreiner V, Schwanitz HJ (2002) The acidic milieu of the horny layer: new findings on the physiology and pathophysiology of skin $\mathrm{pH}$. Am JClin Dermatol 3(4):261-272

13. Czarnowicki T, Krueger JG, Guttman-Yassky E (2017) Novel concepts of prevention and treatment of atopic dermatitis through barrier and immune manipulations with implications for the atopic march. J Allergy Clin Immunol 139(6):1723-1734

14. Wanke I, Steffen H, Christ C, Krismer B, Gotz F, Peschel A et al (2011) Skin commensals amplify the innate immune response to pathogens by activation of distinct signaling pathways. J Invest Dermatol 131(2):382-390

15. Eyerich K, Pennino D, Scarponi C, Foerster S, Nasorri $\mathrm{F}$, Behrendt $\mathrm{H}$ et al (2009) IL-17 in atopic eczema: linking allergen-specific adaptive and microbialtriggered innate immune response. J Allergy Clin Immunol 123(1):59-66

16. Clark RA (2010) Skin-resident T cells: the ups and downs of on site immunity. J Invest Dermatol 130(2):362-370

17. Nakatsuji T, Chen TH, Narala S, Chun KA, Two AM, Yun T et al (2017) Antimicrobials from human skin commensal bacteria protect against Staphylococcus aureus and are deficient in atopic dermatitis. Sci Transl Med 9(378). https://doi.org/ 10.1126/scitranslmed.aah4680

18. Harder J, Dressel S, Wittersheim M, Cordes J, MeyerHoffert U, Mrowietz U et al (2010) Enhanced expression and secretion of antimicrobial peptides in atopic dermatitis and after superficial skin injury. JInvest Dermatol 130(5):1355-1364

19. Werfel T, Allam JP, Biedermann T, Eyerich K, Gilles S, Guttman-Yassky E et al (2016) Cellular and molecular immunologic mechanisms in patients with atopic dermatitis. J Allergy Clin Immunol 138(2):336-349

20. Traidl S, Kienlin P, Begemann G, Jing L, Koelle DM, Werfel Tet al (2018) Patients with atopic dermatitis and history of eczema herpeticum elicit herpes simplex virus-specific type 2 immune responses. J Allergy Clin Immunol 141(3):1144-1147

21. Human Microbiome Project C (2012) A framework for human microbiome research. Nature 486(7402):215-221

22. Grice EA, Segre JA (2011) The skin microbiome. Nat Rev Microbiol 9(4):244-253

23. Kong HH, Andersson B, Clavel T, Common JE, Jackson SA, Olson ND et al (2017) Performing skin microbiome research: a method to the madness. J Invest Dermatol 137(3):561-568

24. Altunbulakli C, Reiger M, Neumann AU, GarzorzStark N, Fleming M, Huelpuesch C et al (2018) Relations between epidermal barrier dysregulation and staphylococci-dominated microbiome dysbiosis in atopic dermatitis. J Allergy Clin Immunol. https://doi.org/10.1016/j.jaci.2018.07.005

25. Zipperer A, Konnerth MC, Laux C, Berscheid A, JanekD, Weidenmaier Cet al (2016) Human commensals producing a novel antibiotic impair pathogen colonization. Nature 535(7613):511-516

26. Byrd AL, Belkaid Y, Segre JA (2018) The human skin microbiome. Nat Rev Microbiol 16(3):143-155

27. Kennedy EA, Connolly J, Hourihane JO, Fallon PG, McLeanWHI, Murray Detal (2017) Skin microbiome before development of atopic dermatitis: early colonization with commensal staphylococci at 2 months is associated with a lower risk of atopic dermatitis at 1 year. J Allergy Clin Immunol 139(1):166-172

28. Meylan $P$, Lang $C$, Mermoud $S$, Johannsen $A$, Norrenberg S, Hohl D et al (2017) Skin colonization by staphylococcus aureus precedes the clinical diagnosis of atopic dermatitis in infancy. J Invest Dermatol 137(12):2497-2504

29. Thorsteinsdottir S, Thyssen JP, Stokholm J, Vissing NH, Waage J, Bisgaard H (2016) Domestic dog 
exposure at birth reduces the incidence of atopic dermatitis. Allergy 71(12):1736-1744

30. Ahmadizar F, Vijverberg SJH, Arets HGM, de Boer A Lang JE, Garssen J et al (2018) Early-life antibiotic exposure increases the risk of developing allergic symptoms later in life: a meta-analysis. Allergy 73(5):971-986

31. Harada K, Saito M, Sugita T, Tsuboi R (2015) Malassezia species and their associated skin diseases. J Dermatol 42(3):250-257

32. Glatz M, Bosshard PP, Hoetzenecker W, SchmidGrendelmeier $P$ (2015) The role of Malassezia spp. in atopic dermatitis. JClin Med 4(6):1217-1228

33. Hannigan GD, Zheng $Q$, Meisel JS, Minot SS, Bushman FD, Grice EA (2017) Evolutionary and functional implications of hypervariable loci within the skin virome. PeerJ 5:e2959

34. El-Heis S, Crozier SR, Healy E, Robinson SM, Harvey NC, Cooper C et al (2017) Maternal stress and psychological distress preconception: association with offspring atopic eczema at age 12 months. Clin Exp Allergy 47(6):760-769

35. Harter K, Hammel G, Krabiell L, Linkohr B, Peters A Schwettmann L et al (2019) Different psychosocial factors are associated with seasonal and perennial allergies in adults - cross-sectional results of the KORA FF4 study. Int Arch Allergy Immunol. https:// doi.org/10.1159/000499042

36. Orivuori L, Mustonen K, de Goffau MC, Hakala S, Paasela M, Roduit C et al (2015) High level of fecal calprotectin at age 2 months as a marker of intestinal inflammation predicts atopic dermatitis and asthma by age 6. Clin Exp Allergy 45(5):928-939

37. Byrd AL, Deming C, Cassidy SKB, Harrison OJ $\mathrm{Ng}$ WI, Conlan S et al (2017) Staphylococcus aureus and Staphylococcus epidermidis strain diversity underlying pediatric atopic dermatitis. Sci Transl Med 9(397). https://doi.org/10.1126/ scitranslmed.aal4651

38. Myles IA, Earland NJ, Anderson ED, Moore IN, Kieh MD, Williams KW etal (2018) First-in-human topical microbiome transplantation with Roseomonas mucosa for atopic dermatitis. JCI Insight 3(9) https://doi.org/10.1172/jci.insight.120608 